\title{
Extended gas-phase trapping followed by surface-induced dissociation of non- covalent protein complexes
}

Sophie R. Harvey ${ }^{1,2}$, Jing Yan ${ }^{1}$, Jeffery M. Brown ${ }^{3}$, Emmy Hoyes ${ }^{3}$ and Vicki H. Wysocki ${ }^{{ }^{*}}$.

1. Department of Chemistry and Biochemistry, Ohio State University, Columbus, Ohio 43210, United States

2. School of Chemistry, Manchester Institute of Biotechnology, University of Manchester, Manchester, M1 7DN, United Kingdom

3. Waters Corporation, Altrincham Road, Wilmslow, SK9 4AX, United Kingdom.

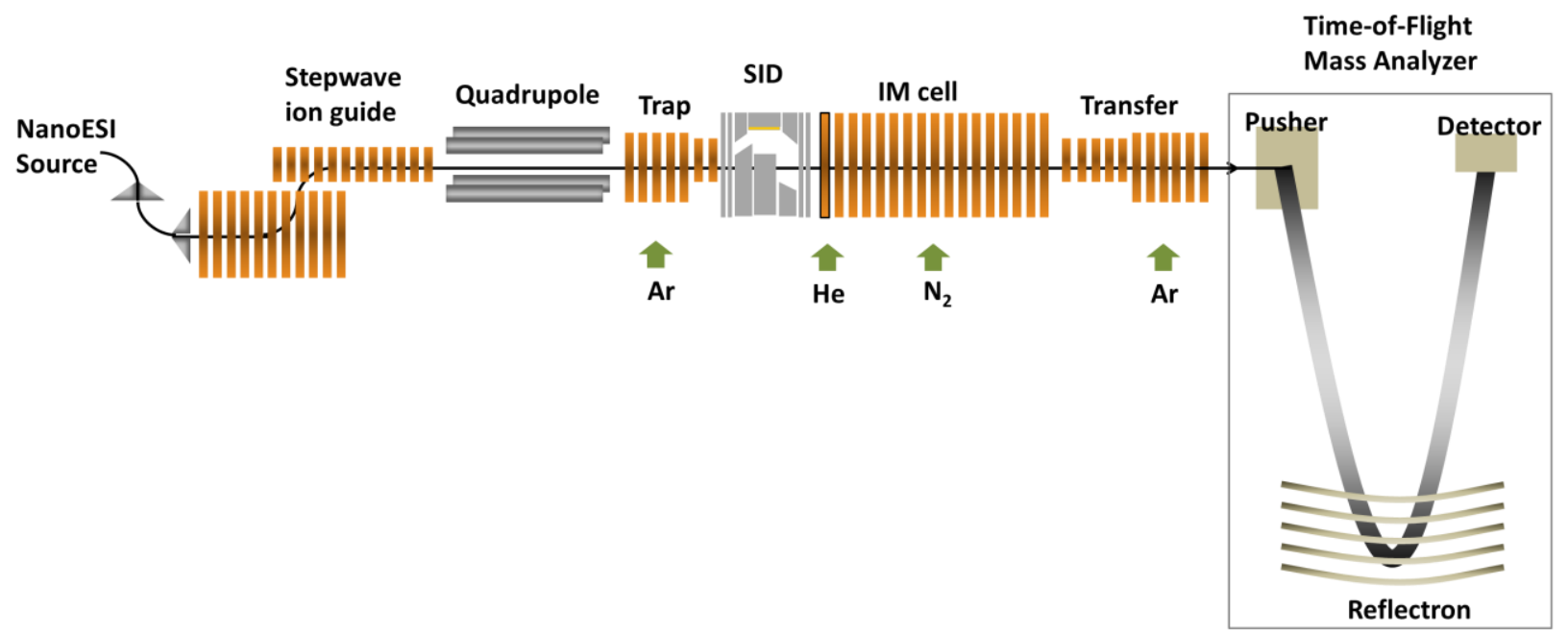

Figure S1: A schematic representation of the in-house modified Synapt G2S mass Spectrometer ${ }^{1}$ used for studies here. In brief, the Trap T-wave region was truncated to enable an SID device to be placed before the ion mobility cell. In this configuration ions trapped within the Trap region can subsequently be fragmented using SID. 


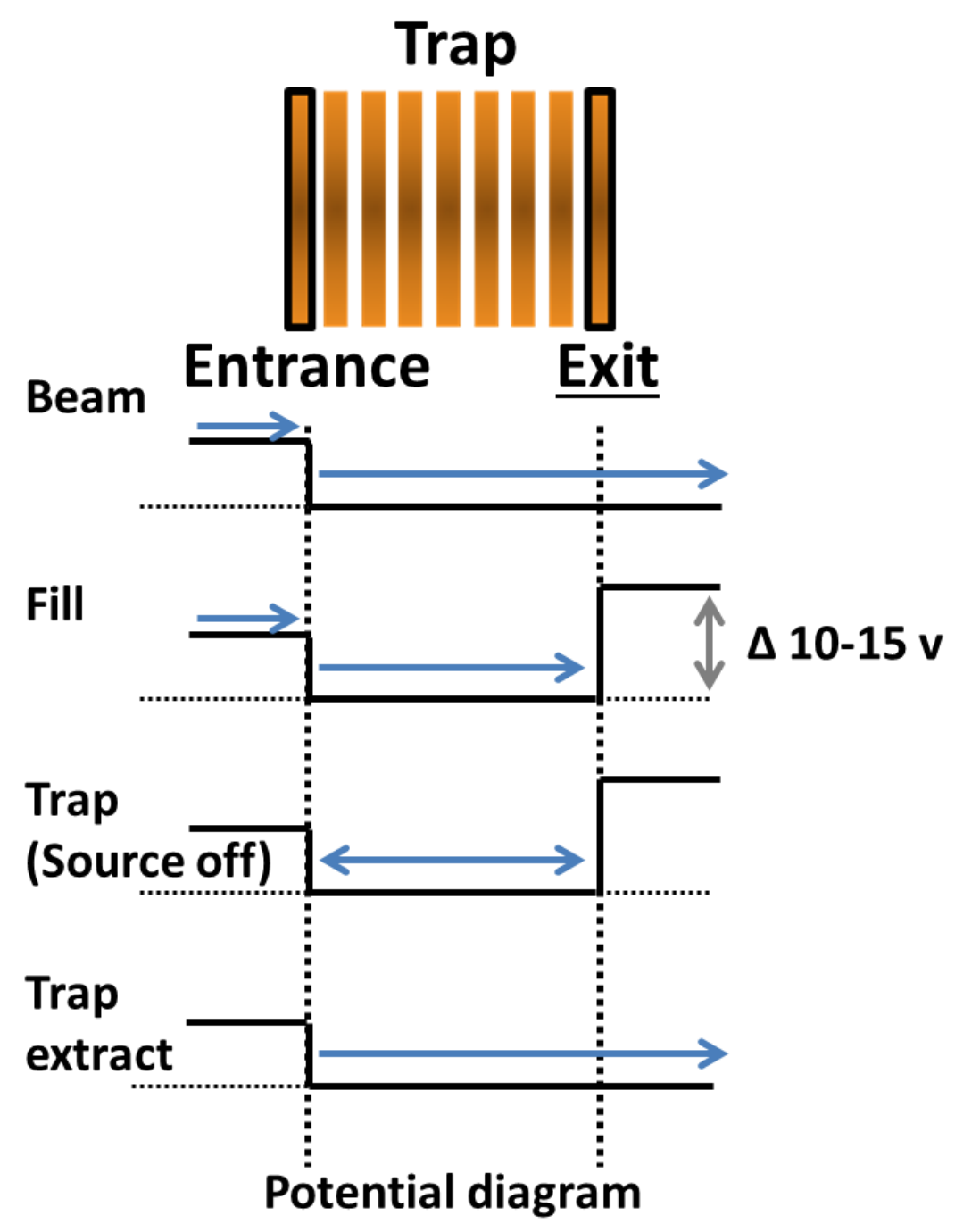

Figure S2: A schematic representation of the trapping process in the Synapt G2S.

\begin{tabular}{lccc}
\hline & $\begin{array}{c}\text { Molecular weight / } \\
\text { kDa }\end{array}$ & $\begin{array}{c}\text { Stopping potential / } \\
\text { V }\end{array}$ & $\begin{array}{c}\text { Trap gas flow rate / } \\
\mathbf{~ m L / m i n ~}\end{array}$ \\
\hline B-lactoglobulin monomer (8+) & 18 & 10 & 1.5 \\
B-lactoglobulin dimer (13+) & 36 & 10 & 2.1 \\
Streptavidin (15+) & 53 & 15 & 2.5 \\
Enolase (19+) & 93 & 10 & 2.5 \\
Concanavilin (21+) & 103 & 15 & 2.7 \\
C-reactive protein (18+) & 115 & 10 & 2.7 \\
Pyruvate kinase (32+) & 237 & 15 & 2.5 \\
\hline
\end{tabular}

Table S1: Stopping potentials and Trap gas flow rates used for all proteins studied here. Values were optimized to obtain the highest trapping efficiency while using the lowest gas flow and stopping potential. 


\begin{tabular}{|c|c|c|c|c|c|c|c|}
\hline & $\begin{array}{l}\text { Molecular } \\
\text { weight / } \\
\text { kDa }\end{array}$ & $1 \mathrm{~s}$ & $5 s$ & $10 \mathrm{~s}$ & $20 \mathrm{~s}$ & $30 \mathrm{~s}$ & $60 \mathrm{~s}$ \\
\hline $\begin{array}{l}\beta \text {-lactoglobulin } \\
\text { monomer (8+) }\end{array}$ & 18 & $86 \pm 5$ & $86 \pm 6$ & $79 \pm 9$ & $70 \pm 14$ & $75 \pm 10$ & $59 \pm 9$ \\
\hline $\begin{array}{l}\beta \text {-lactoglobulin } \\
\text { dimer }(13+)\end{array}$ & 36 & $68 \pm 12$ & $69 \pm 6$ & $57 \pm 13$ & $69 \pm 6$ & $55 \pm 10$ & $50 \pm 3$ \\
\hline $\begin{array}{l}\text { Streptavidin } \\
(15+)\end{array}$ & 53 & $83 \pm 15$ & $85 \pm 6$ & $72 \pm 10$ & $66 \pm 15$ & $74 \pm 3$ & $70 \pm 6$ \\
\hline Enolase (19+) & 93 & $63 \pm 9$ & $54 \pm 10$ & $62 \pm 9$ & $51 \pm 13$ & $59 \pm 4$ & $61 \pm 20$ \\
\hline $\begin{array}{l}\text { Concanavilin } \\
(21+)\end{array}$ & 103 & $70 \pm 16$ & $69 \pm 9$ & $67 \pm 13$ & $68 \pm 16$ & $61 \pm 13$ & $39 \pm 1$ \\
\hline $\begin{array}{l}\text { Pyruvate } \\
\text { kinase }(32+)\end{array}$ & 237 & $74 \pm 6$ & $75 \pm 10$ & $63 \pm 10$ & $66 \pm 6$ & $66 \pm 6$ & - \\
\hline
\end{tabular}

Table S2: Percentage trapping efficiency in the full length trap for trapping times 1, 5, 10, 20, 30 and 60 seconds. From the $5 \mathrm{~s}$ beam check the expected intensity following trapping can be determined, as the fill time is defined (1-3 seconds dependent on precursor intensity), the trapping efficiency is then determined as expected intensity/observed intensity, where observed intensity includes both precursor and charge stripped species.

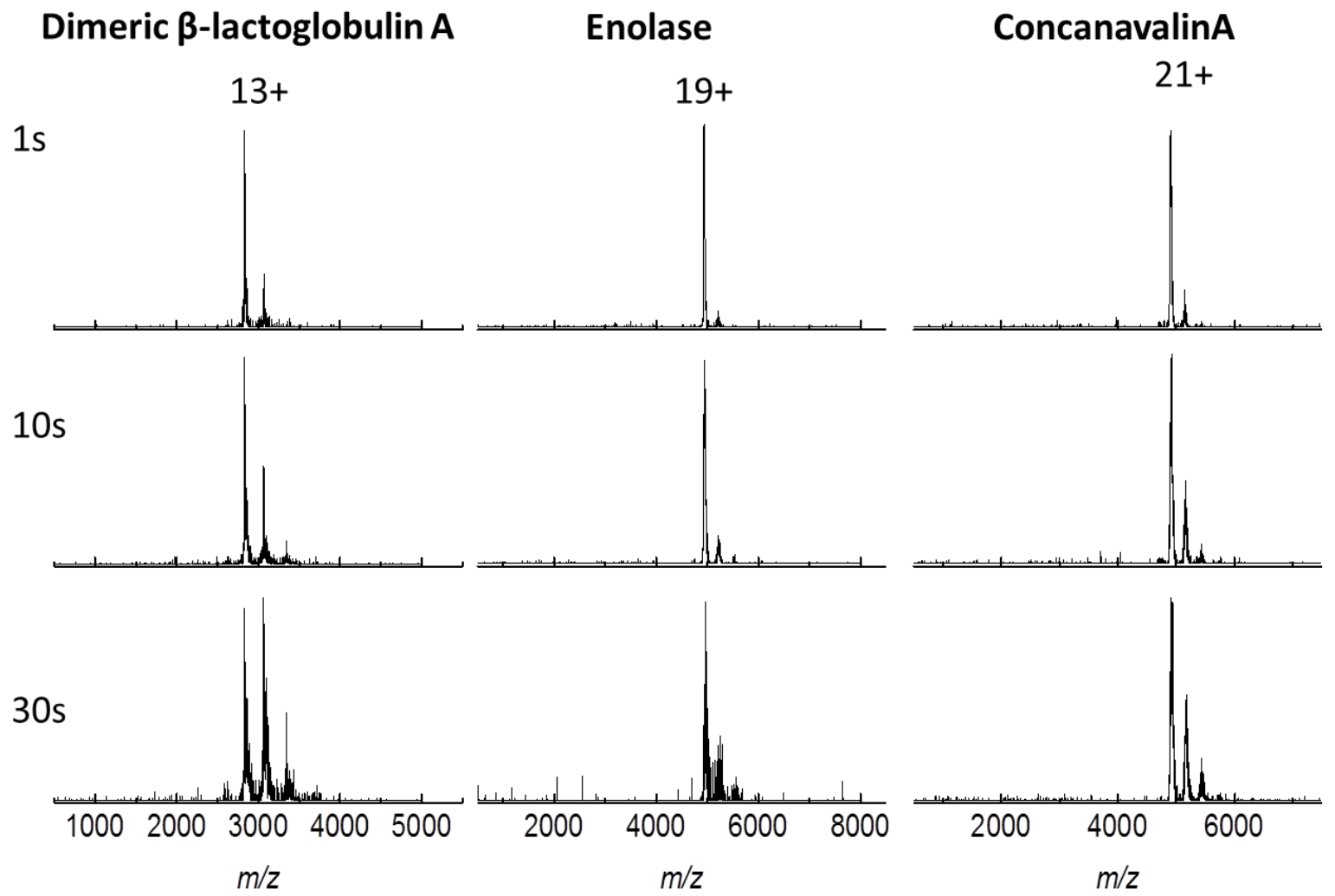

Figure S3: Spectra obtained following trapping for 1, 10 and 30 seconds. Spectra shown are the sum of five extractions pulses. 


\section{Streptavidin}
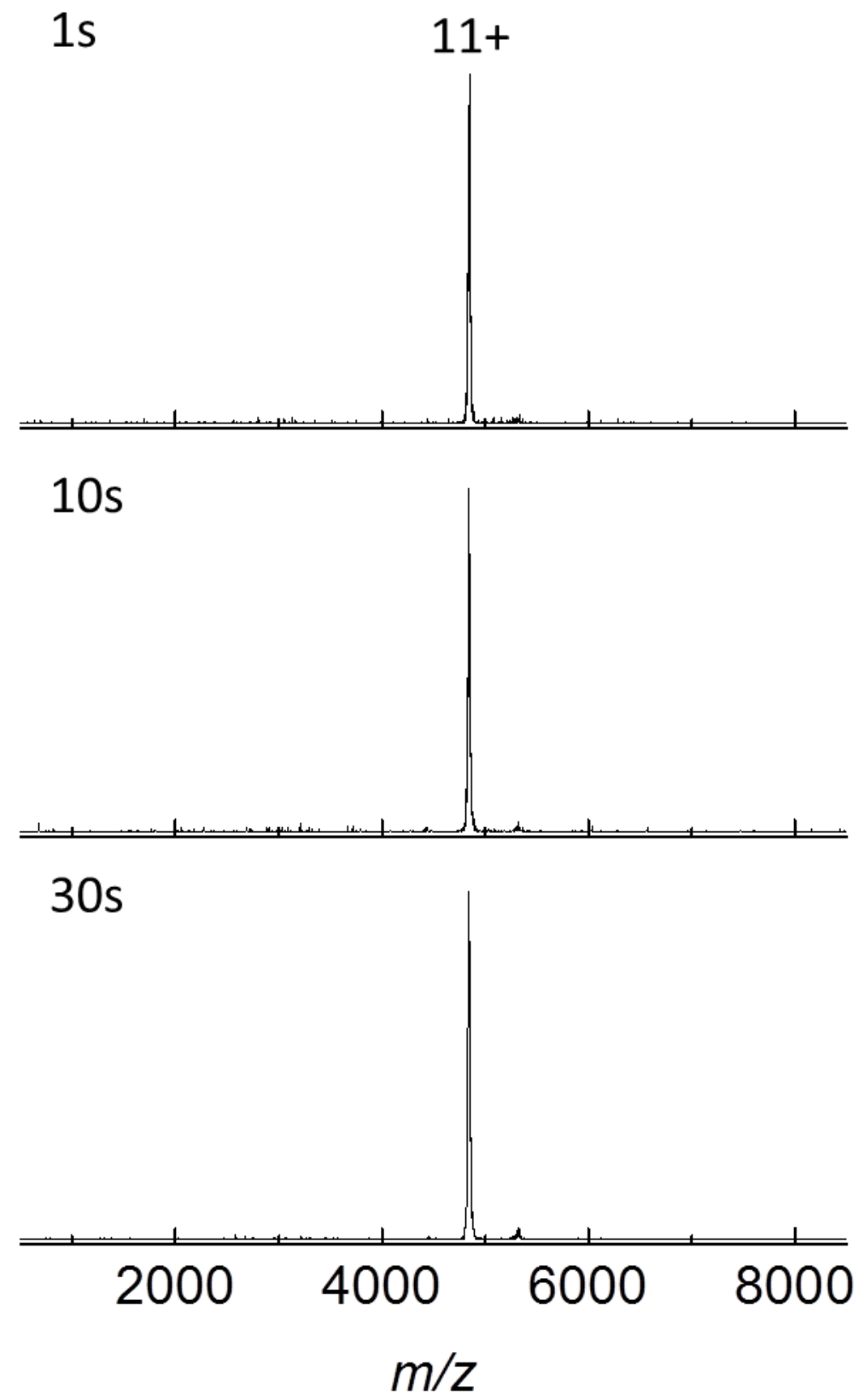

Figure S4: Spectra obtained for 11+ streptavidin following trapping in the truncated trap for 1, 10, or 30 seconds. Spectra shown are the sum of five extractions pulses. 


\begin{tabular}{lllll}
\hline Trapping time/s & Monomer & Dimer & Trimer & Tetramer \\
\hline Beam acquisition & $0.29 \pm 0.02$ & $0.62 \pm 0.01$ & $0.04 \pm 0.01$ & $0.05 \pm 0.02$ \\
1 & $0.29 \pm 0.02$ & $0.60 \pm 0.04$ & $0.04 \pm 0.01$ & $0.07 \pm 0.05$ \\
2 & $0.29 \pm 0.04$ & $0.60 \pm 0.04$ & $0.04 \pm 0.01$ & $0.07 \pm 0.05$ \\
3 & $0.29 \pm 0.05$ & $0.58 \pm 0.06$ & $0.05 \pm 0.02$ & $0.08 \pm 0.05$ \\
4 & $0.28 \pm 0.03$ & $0.61 \pm 0.02$ & $0.04 \pm 0.01$ & $0.07 \pm 0.04$ \\
5 & $0.28 \pm 0.02$ & $0.61 \pm 0.02$ & $0.04 \pm 0.00$ & $0.08 \pm 0.03$ \\
10 & $0.29 \pm 0.02$ & $0.60 \pm 0.03$ & $0.05 \pm 0.01$ & $0.08 \pm 0.03$ \\
15 & $0.29 \pm 0.03$ & $0.58 \pm 0.02$ & $0.05 \pm 0.01$ & $0.08 \pm 0.04$ \\
20 & $0.29 \pm 0.03$ & $0.58 \pm 0.02$ & $0.05 \pm 0.01$ & $0.08 \pm 0.04$ \\
25 & $0.29 \pm 0.07$ & $0.56 \pm 0.02$ & $0.08 \pm 0.05$ & $0.07 \pm 0.05$ \\
30 & $0.28 \pm 0.01$ & $0.56 \pm 0.05$ & $0.08 \pm 0.06$ & $0.08 \pm 0.06$ \\
\hline
\end{tabular}

Table S3: Relative intensity of SID products and remaining precursor, for Streptavidin 11+ beam type SID and following trapping for 1,10 , or 30 seconds. In all cases the SID collision energy was $550 \mathrm{eV}$. Values reported are the average of three repeats and the associated error is given as the standard deviation between repeats. 


\section{Monomer $\bullet$ Dimer $\bullet$ Trimer $\bullet$ Tetramer $\bullet$ Pentamer}
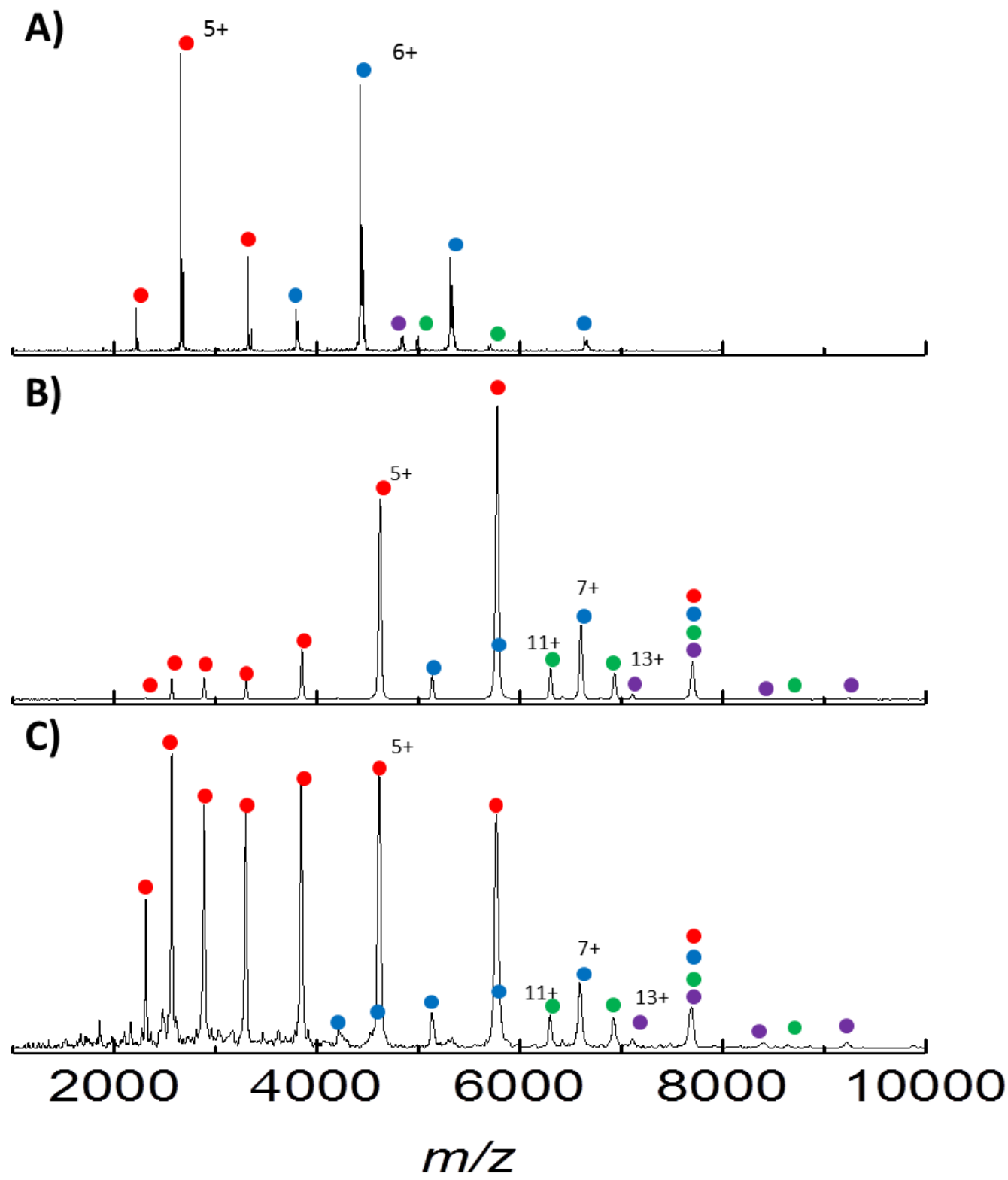

Figure S5: SID spectra obtained from beam acquisition experiments for A) 11+ Streptavidin at a collision energy of $550 \mathrm{eV}$ B) $18+$ C-reactive protein (CRP) pentamer at a SID collision energy of $1440 \mathrm{eV}$ with no cone activation and C) 18+ C-reactive protein (CRP) pentamer at a SID collision energy of $1440 \mathrm{eV}$, using a high cone potential to unfold the complex. Differences in the SID spectra from beam type acquisitions and trapping experiments arise due to the different optic manipulations between these two types of experiments. 


\section{CRP}
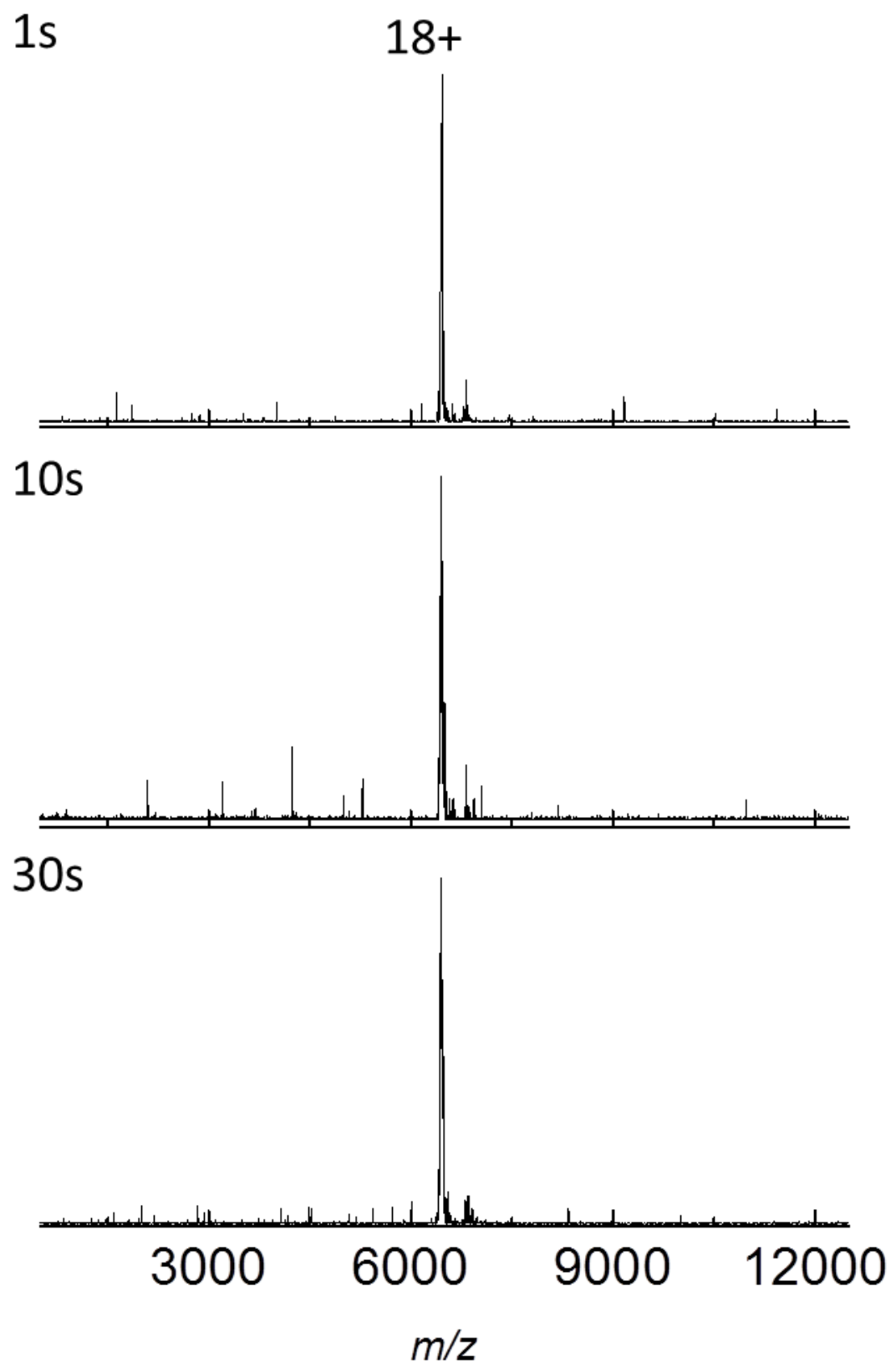

Figure S6: Spectra obtained for 18+ C-reactive protein (CRP) following trapping in the truncated trap for 1,10 , or 30 seconds. Spectra shown are the sum of five extractions pulses 


\begin{tabular}{|c|c|c|c|c|c|}
\hline $\begin{array}{l}\text { Trapping } \\
\text { time/s }\end{array}$ & Monomer & Dimer & Trimer & Tetramer & Pentamer \\
\hline $\begin{array}{l}\text { Beam } \\
\text { acquisition }\end{array}$ & $0.68 \pm 0.01$ & $0.21 \pm 0.01$ & $0.09 \pm 0.01$ & $0.03 \pm 0.01$ & 0 \\
\hline 1 & $0.71 \pm 0.01$ & $0.19 \pm 0.01$ & $0.07 \pm 0.01$ & $0.02 \pm 0.01$ & 0 \\
\hline 2 & $0.72 \pm 0.01$ & $0.19 \pm 0.01$ & $0.07 \pm 0.01$ & $0.02 \pm 0.01$ & 0 \\
\hline 3 & $0.69 \pm 0.02$ & $0.19 \pm 0.01$ & $0.08 \pm 0.02$ & $0.03 \pm 0.01$ & 0 \\
\hline 4 & $0.71 \pm 0.01$ & $0.19 \pm 0.01$ & $0.07 \pm 0.01$ & $0.02 \pm 0.01$ & 0 \\
\hline 5 & $0.71 \pm 0.01$ & $0.20 \pm 0.01$ & $0.07 \pm 0.01$ & $0.02 \pm 0.01$ & 0 \\
\hline 10 & $0.71 \pm 0.01$ & $0.19 \pm 0.01$ & $0.08 \pm 0.01$ & $0.02 \pm 0.01$ & 0 \\
\hline 15 & $0.71 \pm 0.02$ & $0.20 \pm 0.01$ & $0.07 \pm 0.01$ & $0.02 \pm 0.01$ & 0 \\
\hline 20 & $0.71 \pm 0.01$ & $0.19 \pm 0.01$ & $0.07 \pm 0.01$ & $0.02 \pm 0.01$ & 0 \\
\hline 25 & $0.71 \pm 0.01$ & $0.19 \pm 0.01$ & $0.08 \pm 0.01$ & $0.02 \pm 0.01$ & 0 \\
\hline 30 & $0.70 \pm 0.01$ & $0.19 \pm 0.01$ & $0.08 \pm 0.01$ & $0.02 \pm 0.01$ & 0 \\
\hline
\end{tabular}

Table S4: Relative intensity of SID products and remaining precursor, for CRP 18+ beam type SID and following trapping for 1, 10, or 30 seconds. In all cases the SID collision energy was $1440 \mathrm{eV}$. Values reported are the average of three repeats and the associated error is given as the standard deviation between repeats. 


\section{Monomer Dimer}

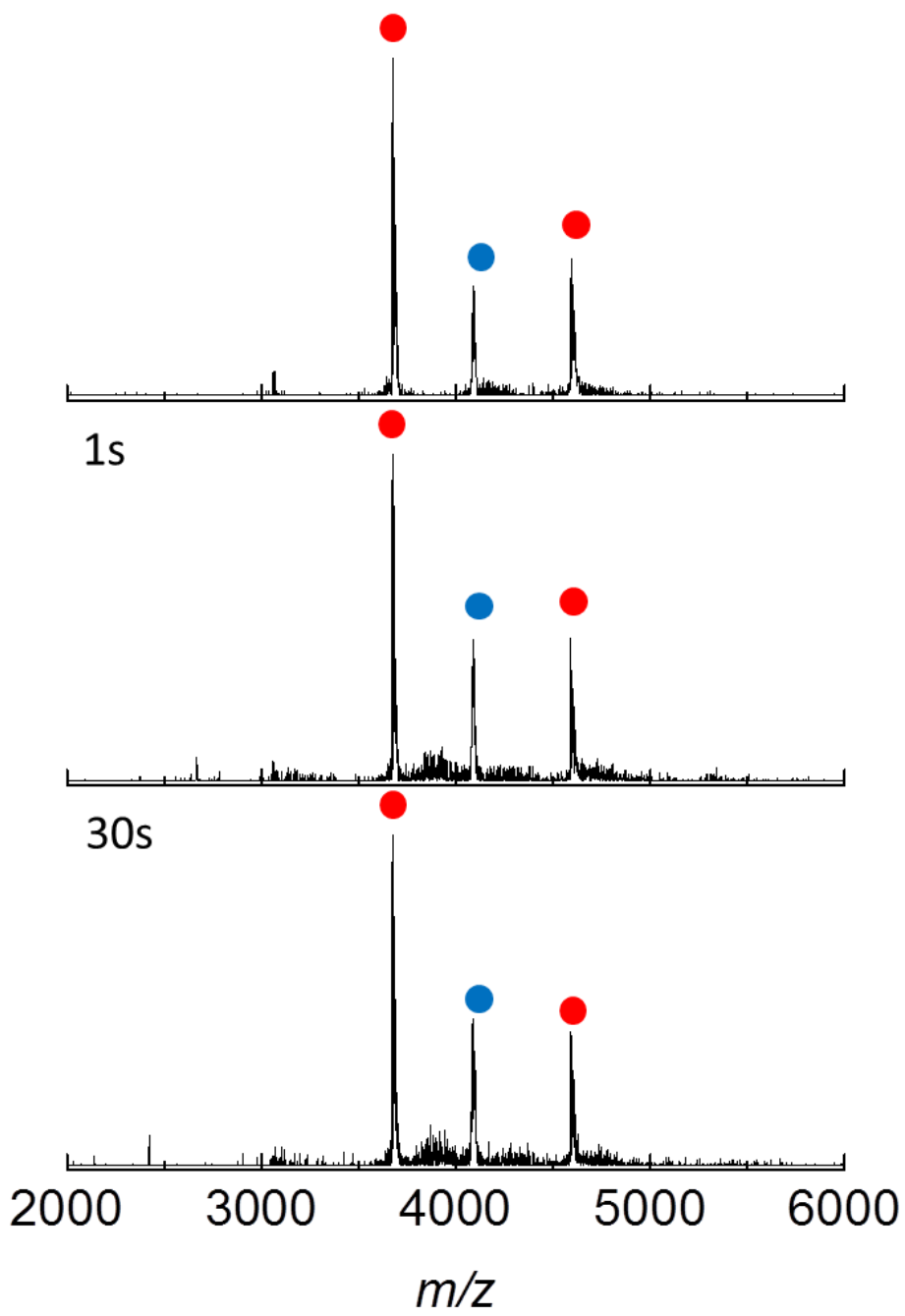

Figure S7: SID Spectra obtained for the charge reduced 9+ $\beta$-lactoglobulin A dimer at a collision energy of $315 \mathrm{eV}$ with no trapping and following trapping for 1 or 30 seconds. Differences in the SID spectra from beam type acquisitions and trapping experiments arise due to the different optic manipulations between these two types of experiments.

\begin{tabular}{lll}
\hline Trapping time/s & Monomer & Dimer \\
\hline Beam acquistion & 0.79 & 0.21 \\
1 & 0.70 & 0.30 \\
10 & 0.69 & 0.31 \\
30 & 0.69 & 0.31 \\
\hline
\end{tabular}

Table S5: Relative intensity of SID products and remaining precursor, for $\beta$-lactoglobulin $9+$ beam type SID and following trapping for 1,10 , or 30 seconds. In all cases the SID collision energy was $315 \mathrm{eV}$. 


\section{Monomer Dimer $\bullet$ Trimer $\bullet$ Tetramer}

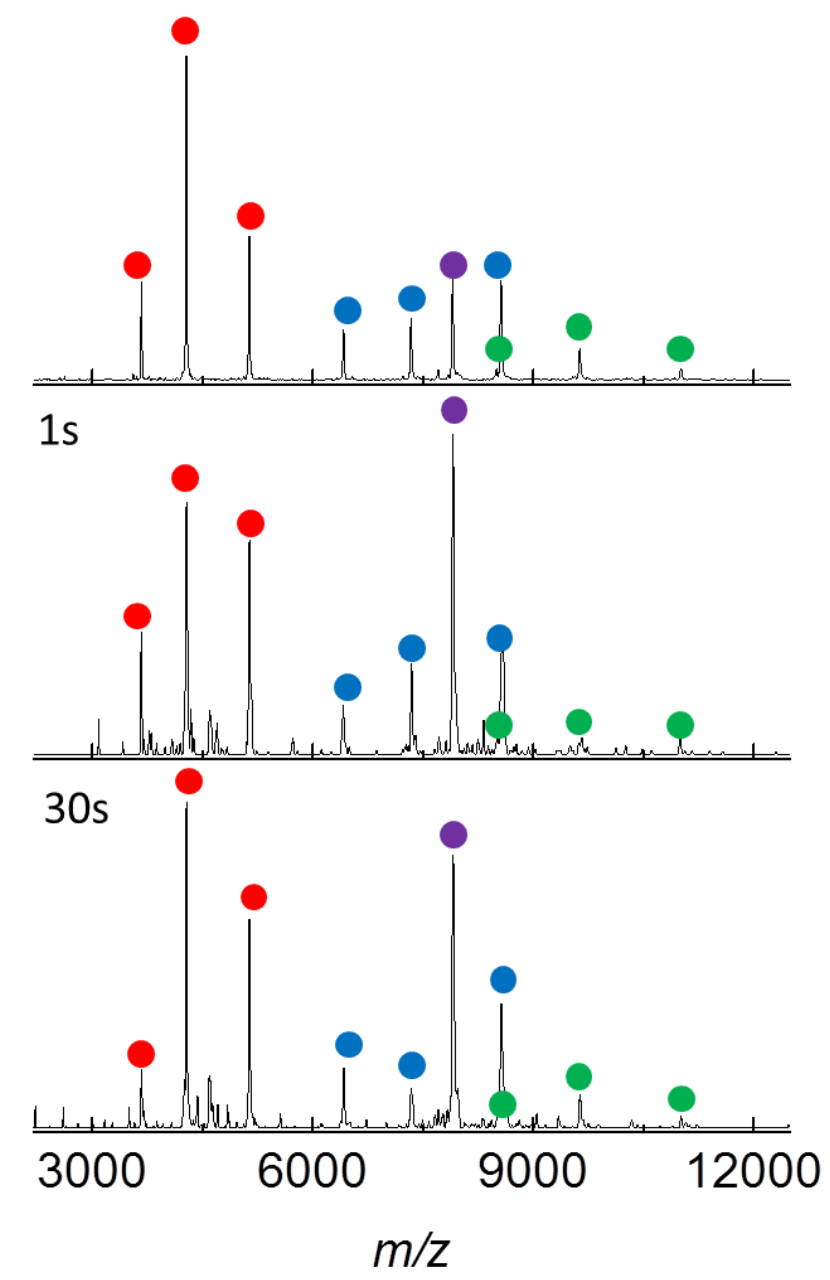

Figure S8: SID Spectra obtained for the charge reduced 13+ concanavilin A tetramer at a SID collision energy of $780 \mathrm{eV}$ with no trapping and following trapping for 1 or 30 seconds. Differences in the SID spectra from beam type acquisitions and trapping experiments arise due to the different optic manipulations between these two types of experiments.

\begin{tabular}{lllll}
\hline Trapping time/s & Monomer & Dimer & Trimer & Tetramer \\
\hline Beam acquisition & 0.57 & 0.23 & 0.08 & 0.11 \\
1 & 0.47 & 0.23 & 0.06 & 0.24 \\
10 & 0.51 & 0.19 & 0.06 & 0.24 \\
30 & 0.44 & 0.21 & 0.07 & 0.27 \\
\hline
\end{tabular}

Table S5: Relative intensity of SID products and remaining precursor, for 13+ concanavilin A beam type SID and following trapping for 1,10 , or 30 seconds. In all cases the SID collision energy was $780 \mathrm{eV}$. A higher relative intensity of remaining precursor is observed after trapping, this could be due to cooling of the ions on trapping. 


\section{References}

1. Zhou M, Huang C, Wysocki VH. Surface-induced dissociation of ion mobility-separated noncovalent complexes in a quadrupole/time-of-flight mass spectrometer. Analytical chemistry. 2012;84(14):6016-6023. 